\title{
Characterization of Wearable and Implanted Antennas: Test Procedure and Range Design
}

\author{
Lukas Berkelmann, Graduate Student Member, IEEE, Dirk Manteuffel, Member, IEEE
}

\begin{abstract}
A method for measuring de-embedded antenna parameters of wearable and implanted antennas for on-body communications is presented. It consists of a tapered flat phantom in order to characterize an antenna's general ability to excite surface waves traveling along the boundary between body tissue and free space, expressed by an angular on-body antenna gain. The design offers a test zone large enough for most typical Wireless Body Area Network devices up to smartphonesize while minimizing the required amount of tissue-simulating material. The designed antenna test range is validated in the 2.4 GHz ISM-band. To showcase the applicability to a realistic application, different designs of antennas integrated into an implanted pacemaker are characterized by their on-body gain patterns. A comparison of their performance in in-situ pathloss measurements reveals a clear relation to the on-body gain patterns and indicates that this parameter is a suitable measure for enabling educated antenna design for on-body applications.
\end{abstract}

Index Terms-Antenna measurements, wireless body area networks, on-body propagation, wearable antennas, implanted antennas

\section{INTRODUCTION}

W IRELESS body area networks (WBAN) enable communication among electronic devices in the vicinity of the body and are established in many areas. The systematic design and characterization of these wireless systems is difficult because of the coupling between the antenna and the human body as part of the propagation channel. In general, we can distinguish between in-body, on-body, and off-body applications. For the off-body case, the IEEE standard test procedures for antennas [1] have already been extended by the CTIA test plan for wireless over-the-air performance [2]. This takes the influence of the user's head and hand on the free-space antenna performance parameters into account. Although the test procedure is more complex because of the use of additional phantoms and predefined test positions for the antenna under test (AUT), the result of the assessment, e.g. the gain pattern, is still well known to the antenna designer and can be used for educated antenna design. In the case of antennas for in-body and on-body wireless links, or respectively combinations of both, the situation is more difficult since the electromagnetic propagation is no longer based on free-space propagation on one hand, and antenna and channel cannot be separated easily on the other hand.

This work was supported by the Deutsche Forschungsgemeinschaft (DFG) under grant MA 4981/11-1.

The authors are with the Institute of Microwave and Wireless Systems, Leibniz University Hannover, Appelstr. 9A, 30167 Hannover, Germany (email: berkelmann@imw.uni-hannover.de, manteuffel@imw.uni-hannover.de)
As a consequence thereof, the common free space radiation patterns are less meaningful for the antenna characterization and design. Therefore, on-body wireless systems so far have been characterized as a whole including transmitter, on-body channel, and receiver (e.g. [3]-[5]). Thus, the antennas are embedded into the channel. Accordingly, measurements with WBAN are usually carried out in-situ with human test subjects or electromagnetic body phantoms. Due to fading and movements of the body, large variations in the path loss can occur making them highly error-prone [5]. Thus, extensive statistical analysis of the measurement results is necessary. Moreover, the measured path loss offers less insight into how the antenna should be designed in general, as it is a function of all system parameters [6].

Recently, different attempts have been made to de-embed on-body channel and antennas with the purpose of deriving de-embedded antenna parameters that enable intuitive insight into the design of on-body antennas. A quite general approach has been proposed by Naganawa et al. [6] using spherical wave functions (SWFs). The coupling between all SWFs at the source and receiver can then numerically be calculated independently of the antennas. Similarly, the antenna can be characterized by its ability to excite those SWFs. However, an implementation of this method for measurements would require the sampling of the antennas near field in the vicinity of the tissue, which seems difficult to realize. Additionally, while mathematically precise, the parameters that can be characterized this way (coefficients of the SWF expansion) cannot be used intuitively in the same way as the otherwise familiar radiation pattern.

Another more specific approach for de-embedding is based on the similarity of the on-body propagation to the so-called Sommerfeld problem of near-earth propagation, where we distinguish between surface waves and space waves. This analogy was discussed in [7] and its applicability to on-body propagation has been investigated in [8], [9].

Recently, we have extended these formulations by defining on-body antenna parameters, such as an angular on-body gain pattern in [10]. The on-body gain pattern establishes a similar metric for on-body propagation as the traditional freespace gain does for classical free-space propagation. In [10], it has been successfully used within a numerical modeling framework for the educated design of antennas for hearing aids and pacemakers.

In this contribution, a test procedure and design of an antenna test range for measuring the on-body radiation pat- 




Fig. 1. On-body communication between wearable and/or implantable antennas based on surface waves (left); modeling/test procedure of approximating the body as a tissue half-space (right)

tern, as defined in [10], is presented. In contrast to the application-specific path loss measurements performed so far, the presented test method for measuring the on-body gain pattern represents a de-embedded, non application-specific, directional measure of an antenna's ability for exciting onbody surface waves. Therefore, in Section II the on-body propagation modeling used for defining the on-body antenna radiation pattern is summarized. For designing the antenna test range, the derivation of the on-body far-field distance is examined as well. Section III explains the general test procedure as well as the design process of the presented antenna test range. The system is designed and validated, but not limited to work, in the $2.4 \mathrm{GHz}$ ISM band, which is most common for current WBAN applications. In Section IV, we prove the applicability of the approach by characterizing antennas for implantable pacemakers. Limitations of the presented approach are discussed in Section V.

\section{On-Body PROPAGATION MOdELING}

Links between on-body WBAN nodes are often non-lineof-sight, e.g. as those depicted in Fig. 1. Therefore, it is well known that TM-mode surface waves traveling along the body surface are a key factor for on-body communication [9], [11]-[13]. These surface waves also represent a main propagation mechanism for links with in-body to in-body, or in-body to on-body propagation with greater separation between the antennas. On-body propagation can be modeled analytically for simplified geometries, e.g. by abstracting the body as a lossy half-space (known as Sommerfeld-problem) [8], see right part in Fig. 1. Alternatively, modeling the body as a cylinder is possible (creeping wave theory, e.g. [12]). For pure surface wave propagation, respectively a normally $(\perp)$ polarized current element located directly on the interface between tissue and air, both problems (flat and curved tissue surface) are closely related, as was shown by Wait [14]. In both cases, the normally polarized E-field $E_{\perp}$ can be described in a form:

$$
E_{\perp}=E_{0} \cdot L_{\mathrm{SW}}
$$

where $L_{\mathrm{SW}}$ is an attenuation function dependent on the dielectric parameters of the tissue and the distance $\rho$ from the source. For the flat tissue half-space, it is calculated by the Sommerfeld attenuation factor $L_{\mathrm{SW}}=F(\rho)$ [14]. In the case of the curved creeping wave problem it also depends on the curvature's radius $q$ : $L_{\mathrm{SW}}=W(\rho, q)$ [14]. The properties of the excitation, i.e. the antenna, are quantified with $E_{0}$. Hence, this factor is to be characterized by the test procedure.

\section{A. Antenna Radiation Pattern for On-Body Communications}

Based on the assumptions that the body surface can be approximated by flat and/or curved parts and on-body propagation primarily relies on surface waves, on-body antenna parameters that describe the ability of an antenna to excite such surface waves can be established. Following the derivation in free space (FS), the on-body (B) antenna gain can be defined as [10]:

$$
G_{\mathrm{B}}(\phi)=\frac{\pi U_{\perp}(\phi)}{P_{\text {in }}|F(\rho)|^{2}} .
$$

Here, the radiation intensity $U_{\perp}=\rho^{2} E_{\perp}^{2}\left(2 \eta_{0}\right)^{-1}$ with normal polarization is measured above an infinite tissue half-space and $P_{\text {in }}$ is the power accepted at the antenna port. For on-body communication along the body surface, the characterization is performed circularly in a plane $(\phi)$ parallel to the tissue half-space rather than on a sphere $(\phi, \theta)$ as in FS. Following this approach, the Friis transmission equation for on-body propagation can be reassembled to [10]:

$$
\frac{P_{\mathrm{r}}}{P_{\mathrm{t}}}=\left(\frac{\lambda L_{\mathrm{SW}}}{4 \pi \rho}\right)^{2} G_{\mathrm{B}, \mathrm{t}} G_{\mathrm{B}, \mathrm{r}},
$$

with $\lambda$ the free-space wavelength. Thus, the characterization of wearable and implantable antennas can be performed at a tissue half-space or a flat phantom, as depicted in Fig. 2. Based on (3), the standard test procedures for the gain measurement of antennas [1] can be adapted for on-body communications.

\section{B. On-Body Far Field}

The on-body gain as defined in (2) is only valid in the far-field of an antenna. This must be ensured for any measurements. In FS the far-field distance is usually derived by limiting the deviation of the incident wave from a plane wave at the test antenna's aperture (Fraunhofer distance) [15]:

$$
\rho_{\mathrm{ff}, \mathrm{FS}} \geq \frac{2 D^{2}}{\lambda},
$$

with $D$ the diameter of the smallest sphere enclosing the antenna.

For on-body propagation, the far field must be redefined since a plane wave approximation is not possible. The approach of the on-body gain, ref. (1), assumes pure surface wave propagation which ideally requires zero-height antenna 


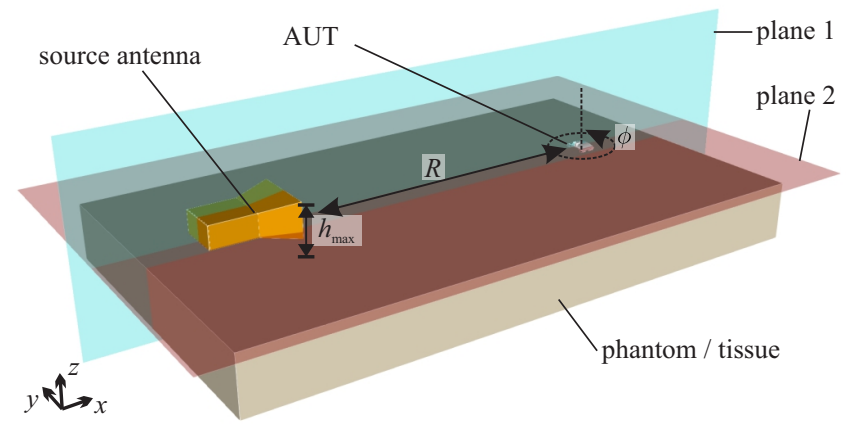

Fig. 2. Measurement principle with planes of analyzed fields.

structures. In [10], we have shown that the observed path loss also with realistic elevated antenna structures can be approximated through the surface wave attenuation term $F(\rho)$ under two conditions:

$$
\rho_{\mathrm{ff}, \mathrm{B}} \geq \sqrt{\left(\frac{16 h z\left|\gamma_{0}\right|}{\pi}\right)^{2}-h^{2}}
$$

and

$$
\rho_{\mathrm{ff}, \mathrm{B}} \geq \frac{z+h}{\left|\Delta_{m}\right|^{2} \sqrt{\varepsilon_{r, 1}}}
$$

where $h$ is the maximum antenna height, $z$ the observer height (see Fig. 1 right), $\gamma_{0}$ the complex propagation constant of free space, $\Delta_{m}=\gamma_{0} / \gamma_{1}$, and $\varepsilon_{\mathrm{r}, 1}$ and $\gamma_{1}$ the relative permittivity and propagation constant of the tissue, respectively. Here, low antenna heights $h, z \ll \rho$ were assumed, which in the following is evaluated in more detail for specifying the test zone for the antenna test range to be designed.

\section{ANTENNA TEST PROCEDURE AND RANGE DESIGN}

Based on the stated principles, an antenna test range for measuring the on-body gain of typical WBAN antennas in the $2.4 \mathrm{GHz}$ ISM-Band is designed. For modeling of the tissue, homogeneous muscle tissue was assumed $\left(\varepsilon_{\mathrm{r}}=52.7, \sigma=\right.$ $1.7 \mathrm{~S} / \mathrm{m}$, for limitations of this simplification see Section V). All numerical simulations were performed using the FDTD solver of EMPIRE XPU [16].

In Fig. 2, the general test procedure is outlined. The transmission coefficient between a feed antenna and the AUT separated by the measurement distance $R$ is measured at a tissue half-space in dependency of the AUT rotation angle $\phi$. As can be derived from the adapted on-body Friss equation (3), the unknown on-body antenna gain $G_{\mathrm{B}, \mathrm{AUT}}$ of the AUT can be calculated from the difference of the transmission coefficient $S_{21}$ compared to a measurement with a known calibration antenna $G_{\mathrm{B}, \text { cal }}$ (all parameters in log-scale /dB) as:

$$
G_{\mathrm{B}, \mathrm{AUT}}=\left|S_{21, \mathrm{AUT}}\right|-\left|S_{21, \mathrm{cal}}\right|+G_{\mathrm{B}, \mathrm{cal}} \text {. }
$$

For both measurements (calibration and AUT), the distance $R$ to the feed antenna is kept constant. Thus, the necessary normalization of the distance-dependent surface wave attenuation factor $F(\rho)$, ref. (2), is achieved inherently. In the following, we will derive the main design parameters of the system.

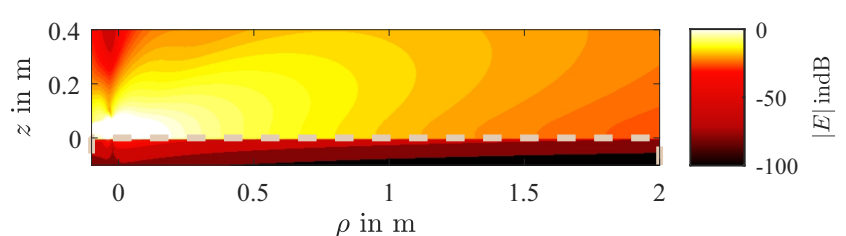

(a)

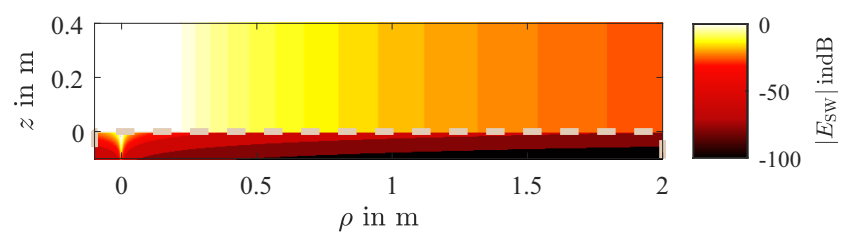

(b)

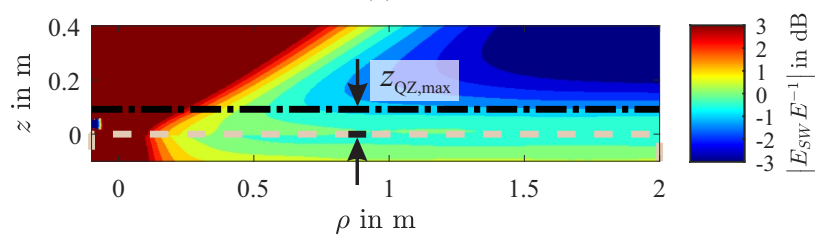

(c)

Fig. 3. (a) Amplitude of the electric field radiated by the horn antenna in plane 1 (ref. Fig. 2) (b) Reference field / Ideal surface wave: $E_{\mathrm{SW}}$, ref. (8)/(9), normalized at $\rho=1000 \mathrm{~mm}, z=1 \mathrm{~mm}$ (c) Deviation from reference field, i.e. QZ. Dashed-dotted line: maximum height $z_{\max }$ calculated for a maximum deviation of $1 \mathrm{~dB}$, dashed line: tissue boundary

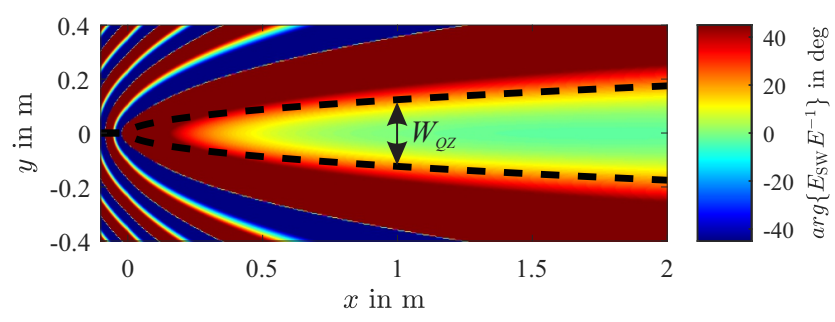

Fig. 4. Phase deviation of the electric field radiated by the horn antenna from surface wave reference $E_{\mathrm{SW}}$ in plane 2 (ref. Fig. 2). Dashed line highlights maximum $\mathrm{QZ}$ width $W_{\mathrm{QZ}}$ for a maximum phase deviation of $22.5^{\circ}$ calculated from (4).

\section{A. Quiet zone estimation}

Standard test ranges are designed such as the uniformity of the illuminating field over a given region within the range meets certain specifications. This region is usually referred to as the quiet zone (QZ) and is the region in which the AUT is to be placed [1]. For FS measurements, the reference is usually a plane wave and accordingly, the QZ is specified by the approximation to a plane wave. In contrast, for measuring the on-body gain (2), the reference is formed by the surface wave, which also defines the on-body far field and can be described in air $(z \geq 0)$ as:

$$
E_{\mathrm{SW}}(\rho, z \geq 0)=E_{0} \cdot F(\rho) e^{-\gamma_{0} \rho} .
$$

Additionally, the electric fields inside the tissue $(z<0)$ can be approximated as:

$$
E_{\mathrm{SW}}(\rho, z<0)=E_{0} \cdot F(\rho) e^{-\gamma_{0} \rho} \gamma_{0}^{2} \gamma_{1}^{-2} e^{\gamma_{1} z} .
$$


The term $\gamma_{0}^{2} \gamma_{1}^{-2}$ was added for fulfilling the boundary condition of the perpendicular E-field at tissue surface, while the term $e^{\gamma_{1} z}$ accounts for the additional losses inside the tissue. Both terms are derived from the on-body Green's functions [10]. The combined definition from (8) and (9) is independent of the feed antenna (height $h$ ) and is thus utilized as the reference field for specifying the QZ.

As illuminating feed antenna, a standard horn antenna (Seavey SGA-30 L, free space gain $G=8.1 \mathrm{dBi}$ at $f=2.45 \mathrm{GHz}$ ), ref. Fig 2 , is used. It is placed at a center height of $h=40 \mathrm{~mm}$ above the tissue with normal polarization towards the tissue surface. Thereby, the highest point of the aperture is at $h_{\max }=70 \mathrm{~mm}$. Fig. 3a depicts the FDTDcalculated amplitude of the electric field radiated by the horn antenna above homogeneous muscle tissue $\left(\varepsilon_{r}=52.7\right.$, $\sigma=1.74 \mathrm{~S} / \mathrm{m}$ ). As can be seen, the fields in plane 1 (perpendicular to the tissue surface, ref. Fig. 2) differ from the reference $E_{\mathrm{SW}}$ depicted in Fig. $3 \mathrm{~b}$. The amplitude of the excited fields is also dependent on the height above the tissue boundary.

Analytically, this height-dependency of the surface wave in air can be approximated under the assumptions $\gamma_{0} \rho \gg 1$ and $\rho^{2} \gg h^{2} ; z^{2}$ through an additional factor $H(h, z)$ [17]:

$$
\begin{gathered}
F(\rho, h, z)=F(\rho) \cdot H(h, z), \\
F(\rho)=1-j \sqrt{\pi K \rho} \cdot w_{\mathrm{F}}(-\sqrt{K \rho}), \\
H(h, z)=\left(1+h \gamma_{0}^{2} / \gamma_{1}\right)\left(1+z \gamma_{0}^{2} / \gamma_{1}\right),
\end{gathered}
$$

with $w_{\mathrm{F}}(x)$ the Faddeeva function and $K=-0.5 \gamma_{0} \Delta_{\mathrm{m}}^{2}$. Considering a constant source height $h$ and limiting the amplitude variation to:

$$
\left|H\left(h, z_{\max }\right) / H(h, z=0)\right|=1+\delta
$$

the maximum height of the QZ can be found as:

$$
z_{\mathrm{QZ}, \max }=\frac{\alpha_{1}+\sqrt{\alpha_{1}^{2}+\left(\delta^{2}+2 \delta\right)\left|\gamma_{1}\right|^{2}}}{|\Delta|^{2}\left|\gamma_{1}\right|^{2}},
$$

with $\alpha_{1}=\operatorname{Re}\left\{\gamma_{1}\right\}$.

With the dielectric parameters of the assumed muscle tissue at $f=2.45 \mathrm{GHz}$, the maximum height is calculated as $z_{\mathrm{QZ}, \max }=92 \mathrm{~mm}$ for limiting the amplitude deviation to $1 \mathrm{~dB}$. Fig. $3 \mathrm{c}$ depicts the amplitude deviation between the excited fields (Fig. 3a) and the surface wave reference (Fig. 3b). As can be seen, the estimation of the maximum height is sufficient for distances above approximately $R \geq 0.5 \mathrm{~m}$.

For minimizing the necessary far-field distance according to (5) and (6), we further limit the highest point of possible antennas under test (AUTs) to $z_{\max }=50 \mathrm{~mm}$, which results in a measurement distance of $R \approx 1000 \mathrm{~mm}$. In plane 2 (ref. Fig. 2), parallel to the tissue surface, the $\mathrm{QZ}$ for the onbody antenna test range is mainly limited by phase variations. Therefore, equivalently to FS, (4) can be used to estimate the width of the QZ, as can be seen in Fig. 4. By rearranging (4) for the AUT diameter $D$ and setting $\rho=R$, we obtain a maximum QZ width of $W_{\mathrm{QZ}, \max }=247 \mathrm{~mm}$ (maximum phase deviation of $22.5^{\circ}$ ).

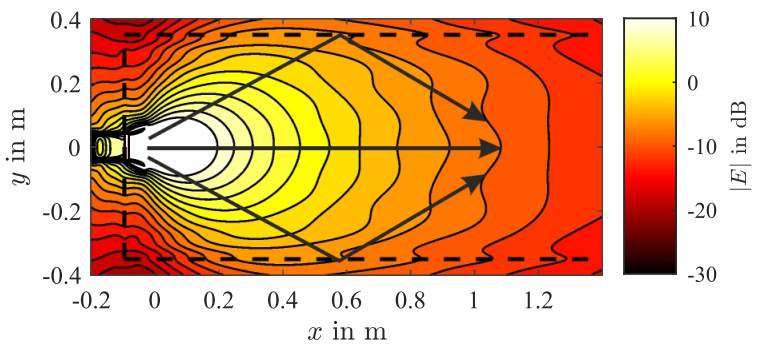

(a)

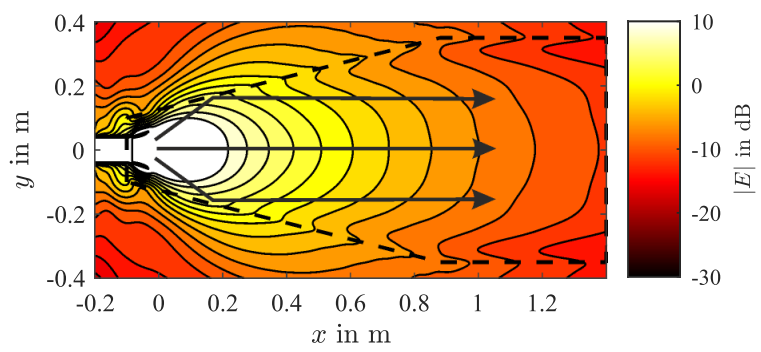

(b)

Fig. 5. FDTD-simulated $E$-field in plane 2 (ref. Fig 2) at $z=20 \mathrm{~mm}$, (a) rectangular body phantom, (b) tapered body phantom

\section{B. Phantom}

As described above, the defined on-body gain, ref. (2), needs to be measured above an infinite tissue half-space. However, a corresponding flat phantom, ref. Fig. 2, can only be realized with finite size which should be kept to a minimum due to the cost and handling of the tissue-simulating material. Size and shape of the flat phantom for the designed measurement setup are optimized by numerical simulations, starting with a rectangular shape, as illustrated exemplarily in Fig. 5a. Here, the flat phantom has a width of $W=0.7 \mathrm{~m}$ and the far end at $x=1.4 \mathrm{~m}$ is terminated with an absorbing boundary. As can be seen, a spatial interference pattern arises through reflections at the phantom edges. A solution for this in form of a tapered shape of the phantom can be adapted from the FS case with tapered anechoic chambers [1], [18]. The taper angle of the flat phantom of $\alpha=23.5^{\circ}$ has been optimized numerically. As illustrated in Fig. 5, the spatial interference pattern varies less and the test zone is illuminated smoothly because of the reduced phase difference between direct propagating waves and the reflected wave at the phantom edge. At the same time, this solution significantly reduces the volume of necessary tissue-simulating material.

\section{Realized setup}

In our setup, we realized the phantom as a Plexiglas tank filled with tissue-simulating liquid as can be seen in Fig. 6. The absorption at the far end is achieved by utilizing a layer of pyramidal absorbers (Emerson \& Cuming WAVASORB VHP-12). For enabling automatic pattern measurements, the AUT needs to be rotated around the axes perpendicular to the tissue surface. To prevent the mechanical parts from coming in contact with the tissue-simulating liquid, we designed a positioner that is supported from above. However, with this setup the bracket holding the AUT is in the line of sight 


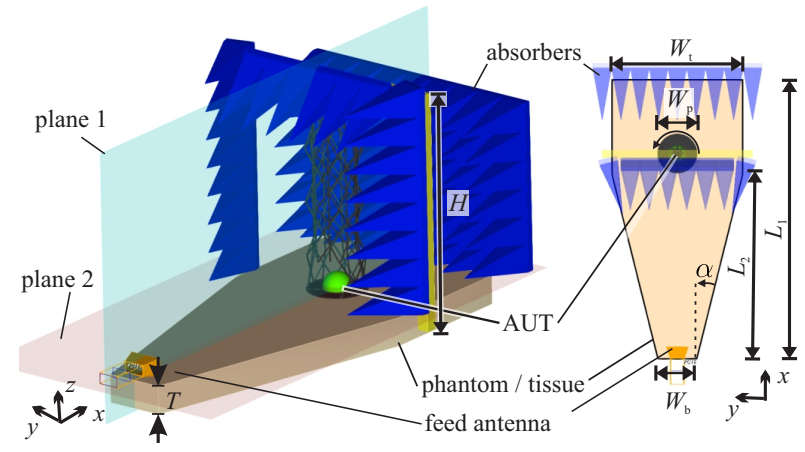

(a)

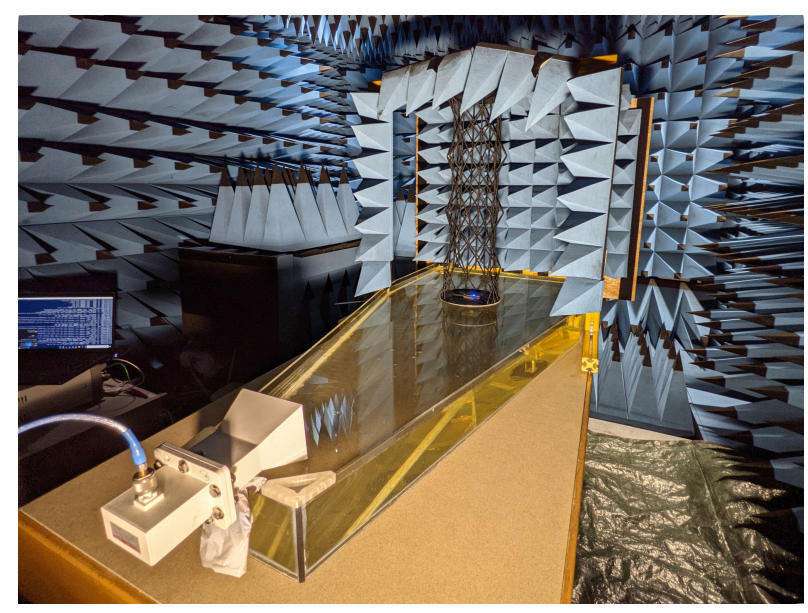

(b)

Fig. 6. On-body antenna test range setup: (a) CAD drawing with dimensions and reference planes, (b) picture of realized system

TABLE I

REALIZED SETUP DIMENSIONS, REF. FIG. 6

\begin{tabular}{cc|cc}
\hline Parameter & Value $(\mathbf{m m})$ & Parameter & Value $(\mathbf{m m})$ \\
\hline \hline$L_{1}$ & 1500 & $W_{\mathrm{p}}$ & 210 \\
$L_{2}$ & 1000 & $H$ & 875 \\
$W_{t}$ & 700 & $\alpha$ & $23.5^{\circ}$ \\
$W_{b}$ & 210 & & \\
\hline
\end{tabular}

between both antennas. Therefore, we optimized the bracket to be as electromagnetically transparent as possible.

As a solution, a cylindrical cage was designed in the form of an iso-truss structure [19], ref. Fig. 6. It is manufactured by $3 \mathrm{D}$ printing of polylactic acid (PLA) plastic $\left(\varepsilon_{r} \approx 3\right)$ and consists of rods with a diameter of $d=5 \mathrm{~mm}$. Numerical simulations revealed that the radar cross-section (RCS) of the designed iso-truss structure is only $12 \%$ of that of a cylinder with $t=2 \mathrm{~mm}$ wall thickness and the same diameter, height and material. Thus, the designed AUT positioner ensures very low reflectivity while maintaining mechanical stability. The frame that supports the iso-truss structure as well as the other mechanical components are covered with pyramidal absorbers. All important dimensions of the realized test range as depicted in Fig. 6 can be found in Table I.

Fig. 7 and 8 depict the results of the numerically evaluated QZ of the complete system. The usable QZ inside the positioner (surrounded by the dash-dotted line) has a horizontal width of $W_{\mathrm{QZ}}=200 \mathrm{~mm}$ and spans in height

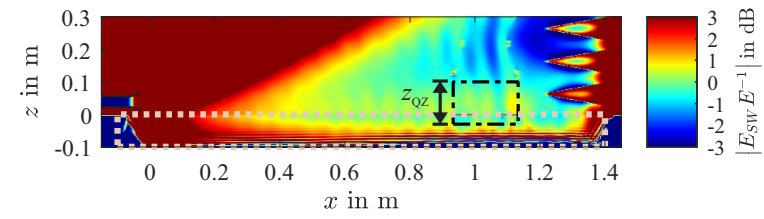

(a)

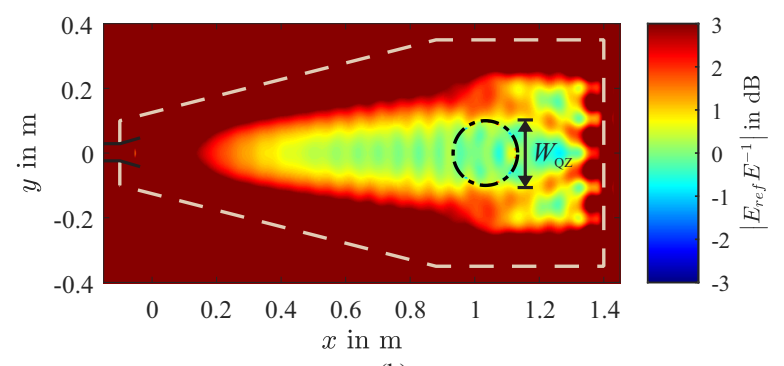

(b)

Fig. 7. QZ: amplitude deviation with final setup: (a) plane 1, ref. Fig. 6; (b) plane 2, usable QZ inside positioner surrounded by dash-dotted line, the dashed line marks the area of the body phantom



(a)

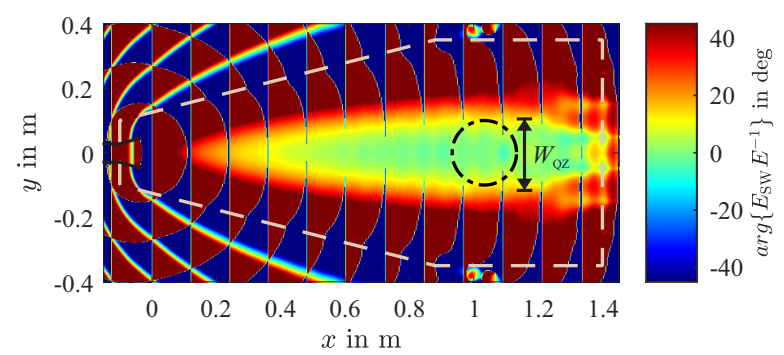

(b)

Fig. 8. QZ: phase deviation with final setup: (a) plane 1, ref. Fig. 6; (b) plane21, test zone surrounded by dash-dotted line, the dashed line marks the area of the body phantom

between $z_{\mathrm{QZ}} \in[-30 \mathrm{~mm} ; 130 \mathrm{~mm}]$. As can be seen in Fig. 7, the amplitude deviation in the test zone of the final setup is $\left|E_{\mathrm{SW}} E^{-1}\right| \leq 1 \mathrm{~dB}$. Also, the maximum phase deviation is $\arg \left\{E_{\mathrm{SW}} E^{-1}\right\} \leq 10^{\circ}$, ref. Fig 8. Thus, overall the determined QZ dimensions correspond closely to the dimensions estimated analytically above. The reflections on the side walls could be reduced well. Nevertheless, the QZ is slightly limited in its width, but this does not have any restrictions for the intended applications. In terms of QZ height above the tissue, the estimate was even slightly exceeded. The small extent in height of the QZ within the tissue is due to the reduced thickness of the phantom, which was chosen to minimize the amount of tissue-simulating fluid required. For typical implant applications like pacemakers, which are most often placed subcutaneously (few centimeters) [20], the achieved test zone depth is still sufficient. 


\section{Validation}

For the measurements in the following, a vertical quarterwave wire monopole $(l=28 \mathrm{~mm})$, centrally positioned on a circular-shaped ground plate (diameter $d=100 \mathrm{~mm}$ ), was used as the calibration antenna. The associated isotropic on-body gain $G_{\mathrm{B}, \text { cal }}=0.25 \mathrm{~dB}$ was determined numerically [10]. The tank was filled with $70 \mathrm{~L}$ of tissue-simulating liquid consisting of a mixture of deionized water with $40 \mathrm{wt} \%$ sucrose [21]. Its dielectric properties were measured using a coaxial probe $\left(\varepsilon_{r}=52.5\right.$ and $\sigma=2.49 \mathrm{~S} / \mathrm{m}$ at $f=2.45 \mathrm{GHz}$ ). The small deviation in conductivity compared to the value used in the simulation only marginally affects the matching of the antennas. The increase in the path loss has no effect on the measured gain results as it is normalized due to the calibration measurement using the same tissue-simulating material.

For validation of the developed setup, we measured the on-body pattern of a half-wave slot dipole antenna, since its simple structure can easily be modeled precisely in the numerical simulation used as a reference for the validation. In the measurement, the antenna's ground plate was oriented perpendicular to the tissue surface, ref. Fig 9a. As can be seen, the antenna is attached to a battery-powered RF module emitting a continuous wave signal at $f=2.45 \mathrm{GHz}$. This way, cable effects due to common mode currents are omitted. The path loss $S_{21, \text { AUT }}$ is calculated from the measured output power of the module and the power received at the feed antenna. Fig. 9b depicts the on-body gain pattern calculated according to the test procedure (7) described above, compared to the pattern obtained from numerical simulation. The deviation for angles $\phi \in\left[90^{\circ} ; 270^{\circ}\right]$ can be explained by the influence of the attached RF module feeding the antenna in the measurement, ref. Fig. 9a. Apart from this, the results of the measurement of the slot dipole antenna with a deviation of $0.3 \mathrm{~dB}$ in peak gain reveal an excellent agreement with the simulated pattern and prove the successful implementation of the measurement principle for the desired on-body gain patterns.

\section{EXAMPLE APPLICATION}

To evaluate the applicability of the proposed test procedure using the flat phantom antenna test range, we analyze the onbody performance of three different antenna structures embedded into a cardiac pacemaker. Since recent pacemakers support Bluetooth in the $2.4 \mathrm{GHz}$ ISM-band for communication with standard consumer devices (e.g. smartphones), on-body propagation becomes an important factor with this application [22]. As can be seen in Fig. 10 a folded dipole, a monopole, and a slot dipole were implemented as example antennas. The antennas are realized on a PCB together with a Bluetoothtransceiver which was then encapsulated in epoxy resin, ref. Fig. 10. The pacemaker housing enclosing the battery and the lower half of the PCB was 3D-printed, electroplated with copper and finished with epoxy resin. The input matching of all three antennas is depicted in Fig. 11. As can be seen each integrated antenna structure is well matched in the band of interest.

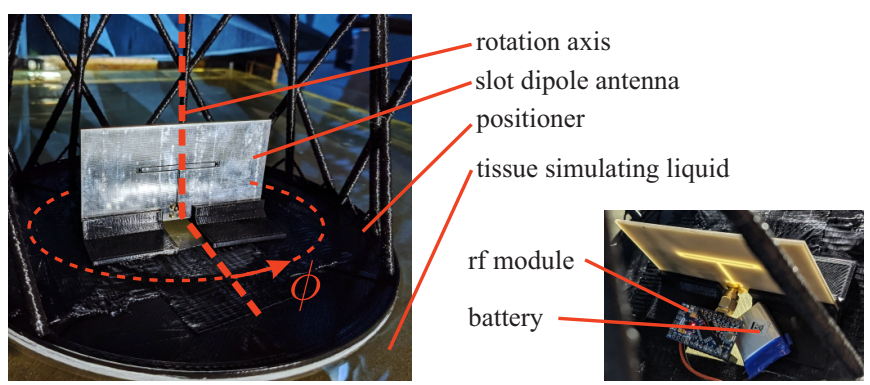

(a)

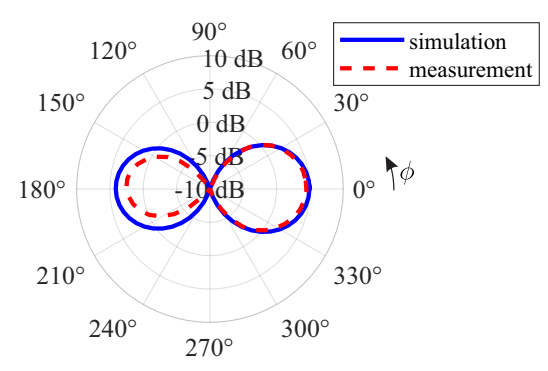

(b)

Fig. 9. Validation measurement: (a) slot dipole antenna with battery-powered RF module inside the positioner, (b) on-body antenna gain pattern of slot dipole antenna

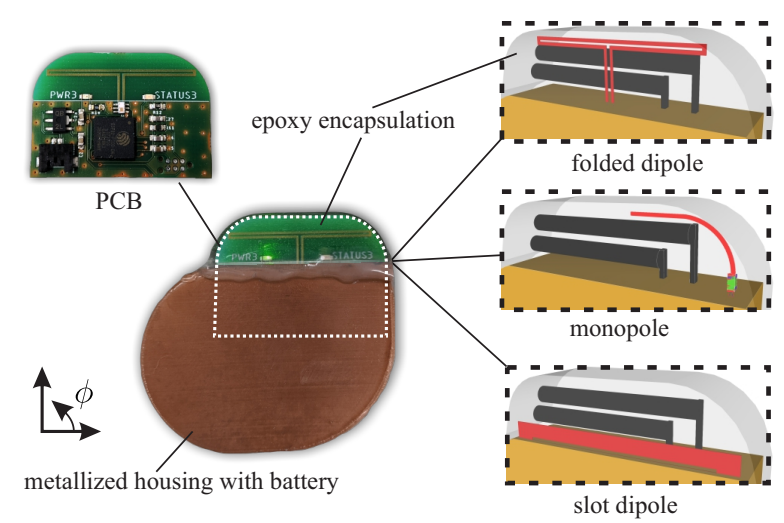

Fig. 10. Cardiac pacemaker example application with three different antenna structures integrated

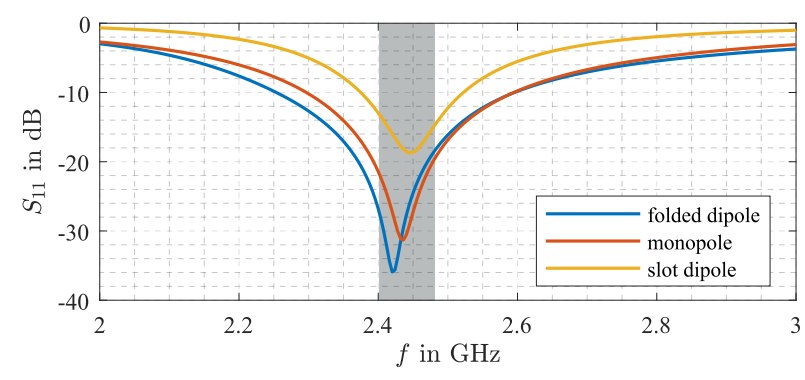

Fig. 11. Input matching of the three antenna structures integrated into the pacemaker. In the simulation, the antennas were implanted at a depth of $h=$ $-20 \mathrm{~mm}$ inside homogeneous muscle tissue. The frequency band of interest is indicated by a grey background. 


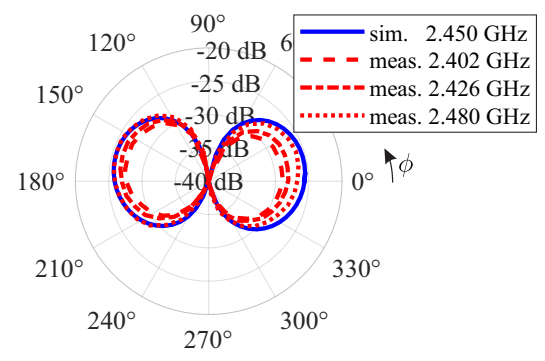

(a) folded dipole



(b) monopole



(c) slot dipole

Fig. 12. Measured on-body gain of pacemaker antennas compared to simulation results.

With the developed antenna test range, the on-body gain of all three antennas placed at a depth of $h=-20 \mathrm{~mm}$ inside the tissue-simulating liquid was measured. Therefore, a spectrum analyzer connected to the feed antenna measured the power of the Bluetooth advertising packets emitted by the Bluetoothtransceivers inside the pacemakers. Hence, results for the on-body gain at the three Bluetooth advertising channels at $f \in[2.042 \mathrm{GHz}, 2.426 \mathrm{GHz}, 2.480 \mathrm{GHz}]$ are gathered. As can be seen in Fig. 12, the measured on-body gain of the antennas agrees reasonably well with the simulated values. The input matching of the antennas during the measurement can also be assumed to agree with the simulation, since the utilized method measures the realized gain and therefore mismatching would reduce the measured gain. The on-body patterns of all three antennas are of similar simple dipole-like shape, but rotated in the diagram resulting in different directions for lobes and nulls. In addition, the peak gain between the different structures differs with the monopole having the lowest peak gain $\left(G_{\mathrm{B}, \max }=-26 \mathrm{~dB}\right)$ and the slot dipole having the highest $\left(G_{\mathrm{B}, \max }=-23.7 \mathrm{~dB}\right)$ of all three antennas. We will come back to these observations later when we discuss the path loss performance of the in-situ tests.
To showcase the insights that the on-body patterns offer, we performed in-situ path loss measurements of the implanted pacemakers communicating with a Bluetooth node (e.g. Smartphone) located at different positions on the body. In order to keep the complexity limited at this point, we used a cylindrical phantom having a diameter of $d=300 \mathrm{~mm}$ and height of $l=1000 \mathrm{~mm}$ for the in-situ tests. The phantom was built from a Plexiglas (thickness $t=4 \mathrm{~mm}$ ) tube filled with tissue-simulating liquid with the same properties as in the onbody gain measurements. The pacemaker antennas were again placed in a depth of $h=-20 \mathrm{~mm}$ inside the liquid. As receiver nodes we used four top-loaded monopole antennas (isotropic on-body gain $G_{B}=1.7 \mathrm{~dB}$, not shown) which were also integrated with a Bluetooth transceiver on a single PCB. Thus, the path loss could be estimated over the air (OTA) with the Bluetooth received signal strength indicator (RSSI). This way, cable effects are avoided. The four reference antennas (ports 25 ) were placed circumferentially around the cylinder with an axial separation of $0.5 \mathrm{~m}$ to the location of the pacemaker, as can be seen in Fig. 13. To account for fading effects, the measurements were repeated five times, whereby each time the antennas were displaced circumferentially by about $\lambda / 8$. Also, the path loss was measured in all three Bluetooth advertising channels and the results were combined for the plot in Fig. 14. Here, the measured value range of the path loss at each position of the reference antennas is displayed as a boxplot. As expected, due to fading, at some positions the measured path loss varies strongly within a range of up to $20 \mathrm{~dB}$. This phenomenon is especially pronounced with antenna 4 which sits at the opposite side of the phantom with respect to the pacemaker, since the length and thus the path loss of the left and right-hand paths of the surface waves is equal here and the two propagation paths lead to a strong fading profile at this position. A meaningful comparison between the different antennas investigated only becomes possible by looking at the mean path loss from multiple measurements, ref. Fig. 14 (marked by circles with dots). Particularly, significant differences can be found with the antennas in positions 2 and 5. Here, the path loss differs by up to $20 \mathrm{~dB}$ in comparison between the different pacemaker antennas.

Now, it is interesting to notice that these effects on the path loss can be easily predicted from the measured on-body gain of the antennas in Fig. 12a-c. Looking at the gain of the folded dipole antenna in Fig. 12a, the poor performance of the link to antenna 2 can be explained by the null of the on-body pattern in the downward direction. The slot dipole has overall the highest gain in the downward direction, therefore the overall observed path loss is the lowest. For the monopole antenna, the gain lobe is directed downward to the left. Thus, the path loss to the left side (position 5) is reduced.

In conclusion, the interpretation above indicates that the onbody gain patterns offer similar insights for the performance of on-body links as free-space patterns do for free-space links, at least if there is a dominant path. Moreover, the example of the folded dipole illustrates that the on-body gain pattern corresponds well with the measured path loss, while the commonly used free-space gain pattern, which has quite the opposite orientation, would obviously lead to wrong 

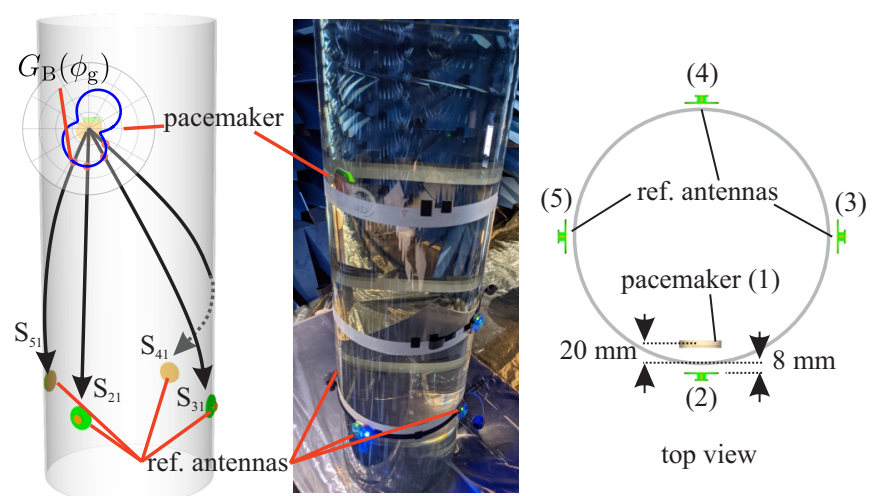

top view

Fig. 13. In-situ measurement setup for the pacemaker example application

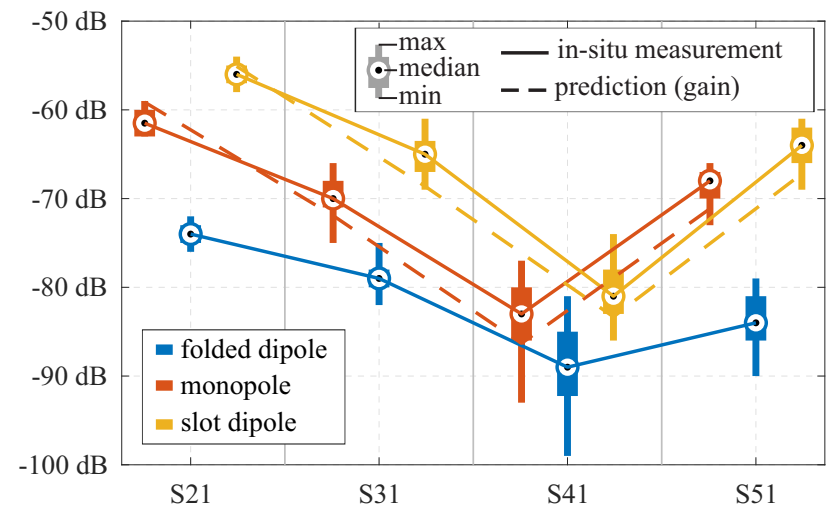

Fig. 14. Results of the path loss measurements as depicted in Fig. 13. Path loss prediction based on the gain difference is calculated according to (15).

assumptions of the on-body path loss.

The illustrative interpretation as discussed above can be quantified for all antennas and all positions in our in-situ example. Based on the assumption that the on-body gain is in average proportional to the overall path loss, we took the measured path loss with the folded dipole antenna as a baseline. From the gain difference in direction of the geodesics to the reference antennas $G_{\mathrm{B}}\left(\phi_{\mathrm{g}}\right)$, see Fig. 13, the path loss of the other two antennas is calculated (all parameters in logscale / dB):

$$
S_{21, \text { pred.,i }}=S_{21, \text { base }}+G_{\mathrm{B}, \mathrm{i}}\left(\phi_{\mathrm{g}}\right)-G_{\mathrm{B}, \mathrm{base}}\left(\phi_{\mathrm{g}}\right),
$$

with $S_{21 \text {,base }}$ and $G_{\mathrm{B} \text {,base }}$ being the measured path loss and on-body gain of the folded dipole antenna, and $G_{\mathrm{B}, \mathrm{i}}$ the measured on-body gain of the monopole or slot dipole antenna, respectively. The results are also plotted in Fig. 14, where the solid lines highlight the mean path losses obtained from the insitu measurements, and the dashed lines depict the prediction from the gain difference added to the path loss of the folded dipole antenna. As can be seen, the overall performance can be predicted well with discrepancies of only a few $\mathrm{dB}$. Also, the ranking of the performance of all three antennas is correct in the prediction at all positions.

\section{LIMITATIONS OF THE APPROACH}

Due to the great variety of propagation phenomena in WBAN, the theory behind our measurement approach requires different assumptions and simplifications. These have already been discussed in detail in [10]. Especially concerning measurements, the tissue modeling should be considered. In our design we considered a phantom simulating a single homogeneous tissue. This simplification has been shown to be accurate at frequencies in the low $\mathrm{GHz}$ range by measurements and simulations [8], [11], [23], [24]. For higher frequencies, multilayered phantoms may be needed. In terms of realization complexity this is another benefit of the proposed measurement approach, as only a flat phantom is needed, compared to the complex body shapes used for in-situ measurements. A two-layer (fat and muscle) flat phantom, as required for the proposed measurement approach, has already been presented in [25]. Furthermore, for any application scenario considered, the body surface in the direct vicinity of the antenna must be sufficiently flat to correctly characterize the on-body gain. Otherwise, protruding body parts, as e.g. the ear for hearing aid applications, can be taken into account as part of the antenna [10]. In the measurement, these need to be physically modeled and added to the positioner.

\section{CONCLUSION}

As discussed, the systematic characterization of antennas for on-body communications is difficult because of the coupling between the antenna and the human body as part of the propagation channel. Analytically, it has been shown in the literature that a de-embedding is possible in the specifically defined on-body far field and that an angular on-body gain can be defined. In this contribution, we have shown how this method can be applied to a test procedure. An antenna test range was designed based on analytical considerations, which should also enable adapting the approach for other applications and frequency ranges. The quiet zone of the designed system has been evaluated numerically, showing only small deviations from the theoretical on-body far field reference fields. The test measurement performed for validating the system revealed an excellent agreement with the simulated pattern, proving the successful implementation of the measurement principle. In order to illustrate the practical applicability of the on-body gain pattern and the related on-body antenna test range for real world applications, a pacemaker mock-up with three different antenna structures was characterized. In addition, the path loss of the implanted pacemaker communicating with multiple receiver nodes distributed on a cylindrical phantom was measured as a simplified example of an in-situ test. Comparing both measurements, it has been shown that the on-body gain pattern of the antenna enables a clear understanding of the relative performance of the different antennas for different links considered. In conclusion, the measured on-body gain offers similar insight into the optimization of antennas for onbody applications as the common free space gain does for free space applications. The designed antenna test range can be used to evaluate the on-body gain for real-world applications. 


\section{REFERENCES}

[1] "IEEE Standard Test Procedures for Antennas," ANSI/IEEE Std 1491979, pp. 0_1-, 1979.

[2] "Test plan for wireless device over-the-air performance," no. Version 3.8.2, Apr. 2019.

[3] A. Alomainy, Y. Hao, A. Owadally, C. G. Parini, Y. Nechayev, C. C. Constantinou, and P. S. Hall, "Statistical Analysis and Performance Evaluation for On-Body Radio Propagation With Microstrip Patch Antennas," IEEE Trans. Antennas Propag., vol. 55, no. 1, pp. 245-248, Jan. 2007.

[4] S. Benaissa, D. Plets, E. Tanghe, G. Vermeeren, L. Martens, B. Sonck, F. A. M. Tuyttens, L. Vandaele, J. Hoebeke, N. Stevens, and W. Joseph, "Characterization of the on-body path loss at 2.45 ghz and energy efficient wban design for dairy cows," IEEE Trans. Antennas Propag., vol. 64, pp. 4848-4858, 2016.

[5] Z. H. Hu, Y. I. Nechayev, P. S. Hall, C. C. Constantinou, and Y. Hao, "Measurements and Statistical Analysis of On-Body Channel Fading at 2.45 GHz," IEEE Antennas Wireless Propag. Lett., vol. 6, pp. 612-615, 2007.

[6] J. Naganawa, J. Takada, T. Aoyagi, and M. Kim, "Antenna Deembedding in WBAN Channel Modeling Using Spherical Wave Functions," IEEE Trans. Antennas Propag., vol. 65, no. 3, pp. 1289-1300, Mar. 2017.

[7] P. S. Hall and Y. Hao, Antennas and Propagation for Body-Centric Wireless Communications, Second Edition. Artech House, 2012.

[8] M. Grimm and D. Manteuffel, "Norton surface waves in the scope of body area networks," IEEE Trans. Antennas Propag., vol. 62, no. 5, pp. 2616-2623, May 2014.

[9] M. Grimm and D. Manteuffel, "On-body antenna parameters," IEEE Trans. Antennas Propag., vol. 63, no. 12, pp. 5812-5821, Dec. 2015.

[10] L. Berkelmann and D. Manteuffel, "Antenna Parameters for On-Body Communications with Wearable and Implantable Antennas," IEEE Trans. Antennas Propag., 2021.

[11] A. Lea, P. Hui, J. Ollikainen, and R. G. Vaughan, "Propagation Between On-Body Antennas," IEEE Trans. Antennas Propag., vol. 57, no. 11, pp. 3619-3627, Nov. 2009.

[12] K. Ali, F. Keshmiri, A. Brizzi, Y. Hao, and C. Craeye, "Body area networks at radio frequencies: Creeping waves and antenna analysis," Comptes Rendus Physique, vol. 16, no. 9, pp. 789-801, Nov. 2015.

[13] N. P. B. Kammersgaard, S. H. Kvist, J. Thaysen, and K. B. Jakobsen, "Ear-to-ear propagation model based on geometrical theory of diffraction," IEEE Trans. Antennas Propag., vol. 67, no. 2, pp. 1153-1160, Feb. 2019

[14] J. R. Wait, "The ancient and modern history of EM ground-wave propagation," IEEE Antennas Propag. Mag., vol. 40, no. 5, pp. 7-24, Oct. 1998.

[15] C. A. Balanis, Antenna Theory: Analysis and Design. USA: Wiley, Apr. 2016.

[16] “EMPIRE XPU Software." [Online]. Available: http://www.empire.de

[17] J. R. Wait, "The Electromagnetic Fields of a Horizontal Dipole in the Presence of a Conducting Half-Space," Canadian Journal of Physics, vol. 39, no. 7, pp. 1017-1028, Jul. 1961.

[18] H. King, F. Shimabukuro, and J. Wong, "Characteristics of a tapered anechoic chamber," IEEE Trans. Antennas Propag., vol. 15, no. 3, pp. 488-490, May 1967.

[19] L. R. Francom and D. W. Jensen, "Three-dimensional iso-tross structure," US Patent US5 921 048A, Jul. 13, 1999.

[20] A. Johansson, "Wireless Communication with Medical Implants: Antennas and Propagation," Department of Electroscience, Lund University, Jan. 2004

[21] S. Castell-Palacios, A. Valls-Lluch, C. Garcia-Pardo, A. Fornes-Leal, and N. Cardona, "Formulas for easy-to-prepare tailored phantoms at 2.4 GHz ISM band," in 2017 11th International Symposium on Medical Information and Communication Technology (ISMICT), Feb. 2017, pp. $27-31$

[22] P. R. Roberts and M. H. ElRefai, "The Use of App-based Follow-up of Cardiac Implantable Electronic Devices," Cardiac Failure Review, vol. 6, Apr. 2020.

[23] J. Liu, Y. Shao, J. Luo, P. Wang, and J. Zhang, "On-Body Channel Characteristics Based on Variation of Human Tissue Properties," in 2019 International Symposium on Antennas and Propagation (ISAP), Oct. 2019, pp. 1-3.

[24] F. Keshmiri and C. Craeye, "Moment-method analysis of normal-tobody antennas using a green's function approach," IEEE Trans. Antennas Propag., vol. 60, no. 9, pp. 4259-4270, Sep. 2012.
[25] M. Barbi, C. Garcia-Pardo, A. Nevrez, V. Pons Beltrn, and N. Cardona, "UWB RSS-Based Localization for Capsule Endoscopy Using a Multilayer Phantom and In Vivo Measurements," IEEE Trans. Antennas Propag., vol. 67, no. 8, pp. 5035-5043, Aug. 2019.

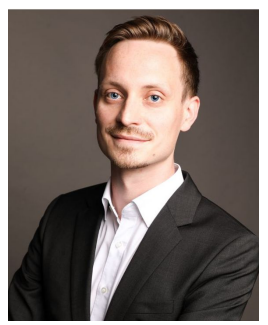

Lukas Berkelmann (GS'17) was born in Stadthagen, Germany, in 1991. He received the B.Sc. and M.Sc. degrees in electrical engineering and information technology from the Leibniz University Hannover, Hannover, Germany, in 2014 and 2016, respectively. Since 2017 he has been a Research Assistant with the Institute of Microwave and Wireless Systems, Leibniz University Hannover. His current research is focused on the modeling of antennas and propagation for body-centric communication and EM modeling for biomedical applications.

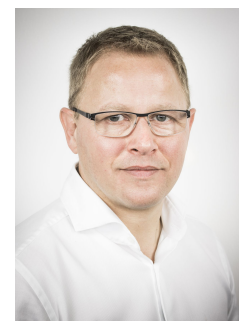

Dirk Manteuffel (M'09) was born in Issum, Germany, in 1970. He received the Dipl.-Ing. and Dr.-Ing. degrees in electrical engineering from the University of Duisburg-Essen, Essen, Germany, in 1998 and 2002, respectively. From 1998 to 2009, he was with IMST, Kamp-Lintfort, Germany. As a Project Manager, he was responsible for industrial antenna development and advanced projects in the eld of antennas and EM modeling. From 2009 to 2016, he was a Full Professor of wireless communications at Christian-Albrechts-University, Kiel, Germany. Since June 2016, he is a Full Professor and the Director of the Institute of Microwave and Wireless Systems, Leibniz University Hannover, Hannover, Germany. His research interests include antenna integration and EM modeling for mobile communications and biomedical applications. Dr. Manteuffel was the recipient of the Young Scientist Award of the Vodafone Foundation for Science in 2004 for his research on the analysis and design of integrated mobile phone antennas with special emphasis on the interaction with the user. 